\title{
Preparation of Highly Concentrated Silver Nanoparticles in Reverse Micelles of Sucrose Fatty Acid Esters through Solid-Liquid Extraction Method
}

\author{
Hidetaka Noritomi*, Yoshihiro Umezawa, Saori Miyagawa, Satoru Kato \\ Department of Applied Chemistry, Tokyo Metropolitan University, Tokyo, Japan \\ E-mail: ${ }^{*}$ noritomi@tmu.ac.jp \\ Recieved August 12, 2011; revised September 16, 2011; accepted September 20, 2011
}

\begin{abstract}
Silver nanoparticles were synthesized in reverse micelles consisting of sucrose fatty acid esters by dissolving reactant powder in the water pool of reverse micelles through the solid-liquid extraction. Silver nanoparticles having various sizes and shapes were obtained at high concentration. The size of silver nanoparticles was controlled by reaction temperature. Moreover, the size of silver nanoparticles was dependent upon the average esterification degree of sucrose fatty acid esters forming reverse micelles. The wavelength in the peaks, which corresponded upon the localized surface plasmon resonance of resultant silver nanoparticles, was correlated with their sizes.
\end{abstract}

Keywords: Reverse Micelle, Silver Nanoparticle, Size Control, Solid-Liquid Extraction, Sucrose Fatty Acid Ester

\section{Introduction}

In recent years, the production and applications of metal nanoparticles have rapidly been increased in the field of nanotechnology, since their novel physical and chemical properties are not only different from those of bulk substances due to their extremely small size and large specific surface area, but also metal nanoparticles exhibit specific colors due to localized surface plasmon resonance (LSPR) corresponding to the wavelength of light that induces the largest electromagnetic field on the nanopartices, when metal nanoparticles are impinged by a beam of light [1]. Especially, the applications of silver nanoparticles have widely been investigated, since they exhibit some profitable properties such as catalysis [2], antibacterial agent [3], nanoparticle colorant [4], nanopaste for electrical circuits and electrodes [5], and substrates for surface-enhanced Raman scattering [6]. The colors of silver nanoparticles result from changes of LSPR induced by their size and shape.

In order to produce metal nanoparticles having suitable sizes and/or shapes, many physical or chemical techniques such as coprecipitation, gas-evaporation, solgel method, and sputtering have been developed so far [1]. Additionally, more attention has been paid on the preparation of metal nanoparticles in reverse micelles [7]. Reverse micelles are thermodynamically stable nanometer-sized aggregates of surfactant molecules dispersed in a hydrophobic organic phase like octane, and can form w/o type microemulsions containing a small amount of water in their centers. The water phase in w/o type microemulsions is called water pool. Metal nanoparticles are synthesized in the water pool, in which reactants are dissolved. As the preparation of metal nanoparticles using reverse micelles does not require a special apparatus and extreme conditions of temperature and pressure, it is comparatively easy to expand the scale of reverse micellar reaction system. However, the productivity of metal nanoparticles per volume of reverse micellar system is limited due to low overall concentration of reactants in the reverse micellar system, since the volumetric ratio of water phase playing a role as the dissolution of reactants and the reaction field to the bulk organic phase is too small [1].

In order to solubilize reactants in reverse micelles, three solubilization methods are utilized: injection method; liquid-liquid extraction method; solid-liquid extraction method [8]. First, the injection method is carried out by injecting a few microliters of the concentrated stock solution of reactants into the hydrocarbon solution 
of surfactants. This method is most commonly used to prepare metal nanoparticles in reverse micelles. Second, the liquid-liquid extraction method is carried out by transferring solutes dissolved in an aqueous phase from the aqueous phase into the hydrocarbon phase of surfacetants. This method is relatively slow, and the concentration of reactants in the hydrocarbon phase is limited due to the distribution between aqueous and reverse micellar organic phases in thermodynamic equilibrium. Third, the solid-liquid extraction method is carried out by mixing solid reactants with the reverse micellar solution containing already a certain amount of water. This method can dissolve reactants until their dissolution reaches saturation in water pool. Moreover, when using the solid-liquid extraction instead of the conventional injecttion method to solubilize reactants into reverse micelles, the following benefits are expected. First, the productiveity of metal nanoparticles is markedly promoted, since reactants consumed for the formation of metal nanoparticles are successively supplied from solid reactants already added in large excess, compared to the solubility of the water pool of reverse micelles. Second, only a small amount of water is needed for solid-liquid extraction. Consequently, the aggregation of resultant nanoparticles is inhibited, since the lower water content is kept in reverse micellar system, the more stably nanoparticles are dispersed [9]. However, there have not been any reports about the preparation of nanoparticles in reverse micelles using the solid-liquid extraction method.

In our previous work, we have reported that silver nanoparticles are prepared in reverse micelles of sucrose fatty acid esters by using the conventional injection method [10]. Sucrose fatty acid esters are commercial food grade nonionic surfactants, and are biodegradable and nonhazardous to the environment [11]. In the present work, we examined the preparation of silver nanoparticles in reverse micelles of sucrose fatty acid esters by using solid-liquid extraction method to address how the preparation conditions such as the reaction temperature and the composition of surfactants affect the size and shape of silver nanoparticles.

\section{Experimental}

Silver nitrate and sodium borohydride were the guaranteed reagents of Kanto Chemicals (Tokyo, Japan). DKSS (sucrose fatty acids of $99 \mathrm{wt} \%$ monoesters and $1 \mathrm{wt} \%$ di- and triesters, fatty acid constituent consisting of 60 wt $\%$ stearic acid and $40 \mathrm{wt} \%$ palmitic acid) and DK-F$20 \mathrm{~W}$ (sucrose fatty acids of $11 \mathrm{wt} \%$ monoesters, $36 \mathrm{wt} \%$ di- and triesters, and $53 \mathrm{wt} \%$ more than tetraesters, fatty acid constituent consisting of $70 \mathrm{wt} \%$ stearic acid and 30 wt\% palmitic acid) were supplied from Dai-Ichi Kogyo
Seiyaku (Kyoto, Japan). The surfactant was used without further purification. Isooctane and n-butanol were from Kanto Chemicals (Tokyo, Japan), and were of analytical grade.

The reverse micellar solutions were prepared by adding the required amounts of sucrose fatty acid esters and a small amount of water into the solution of n-butanol/ isooctane $(3: 7(\mathrm{v} / \mathrm{v}))$, and then were used for the preparation of nanoparticles within a few minutes.

The preparation of nanoparticles was carried out by adding a certain amounts of $\mathrm{AgNO}_{3}$ powder and sodium borohydride powder into the reverse micellar solution.

Since the preliminary study indicated that the synthesis of particles finished at $25^{\circ} \mathrm{C}$ after $3 \mathrm{~h}$, and no residual solid reactants existed in the reaction medium, the samples for various analyses were taken after $3 \mathrm{~h}$. In the present work, the concentrations of sucrose fatty acid esters and water based on the overall volume of reverse micellar solution were fixed at $50 \mathrm{~g} / \mathrm{L}$ and $60 \mathrm{mM}$, respecttively. As a typical condition, the overall concentrations of $\mathrm{AgNO}_{3}$ and sodium borohydride were 0.1 and $1.0 \mathrm{M}$, respectively, and the temperature was fixed at $25^{\circ} \mathrm{C}$.

The TEM micrograph was obtained using a JEOL JEM-2000FX electron microscope operating at $200 \mathrm{kV}$. The sample for TEM was prepared by diluting a resultant colloidal solution one hundred-fold with n-butanol and placing a drop of colloidal solution onto the standard carbon-coated copper grids and drying it under vacuum. The UV-vis spectra of the reverse micellar solutions containing nanoparticles were measured by UV/vis spectrophotometer (Ubest-55, Japan Spectroscopic Co. Ltd.) with a $10 \mathrm{~mm}$ quartz cell.

\section{Results and Discussion}

\subsection{Productivity of Silvar Nanoparticles through Solid-Liquid Extraction Method}

In order to successively supply reactants consumed for the formation of silver nanoparticles to the water pools of reverse micelles, which are reaction fields, and produce silver nanoparticles at high concentration, we have employed solid-liquid extraction method instead of the conventional injection method [8]. The colloidal solution of silver nanoparticles prepared by solid-liquid extraction method was much concentrated, compared to that prepared by the injection method examined in our previous method [10]. The concentration of silver nanoparticles obtained by the present system was about one hundred and thirty times larger than that obtained by the injection method. After diluting the resultant colloidal solution one hundred-fold with n-butanol, the measurement of UV-visible absorption spectrum was carried out. The 
color of the solution after dilution was yellow, similar to the case of injection method, and, as shown in Figure 1, its UV-visible absorption spectrum exhibited the peak around $403 \mathrm{~nm}$, which corresponds upon the LSPR of silver nanoparticles [12]. Figure 2 shows the relationship between surfactant concentration and the absorbance at $403 \mathrm{~nm}$ of colloidal solutions of silver nanoparticles preliminarily diluted one handred-fold with n-butanol. Any absorbance at $403 \mathrm{~nm}$ was not observed without surfactant. On the other hand, the absorbance at $403 \mathrm{~nm}$ increased above $20 \mathrm{~g} / \mathrm{L}$ of surfactant concentration. We have reported that the apparent critical micelle concentration in sucrose fatty acid/n-butanol/isooctane system was observed around $10 \mathrm{~g} / \mathrm{L}$ [13]. Consequently, it is suggested that the preparation of silver nanoparticles proceeds as follows. First, empty reverse micelles containing a small amount of water approach solid reactants added in large excess, compared to the solubility of the water pool of reverse micelles. When the water pools of reverse micelles contact solid reactants, reactants are solubilized into the water pools. Then, silver nanoparticles are synthesized from reactants solubilized in reverse micelles. Resultant silver nanoparticles are coated by surfactants, and are dispersed in organic solvents. Emp-

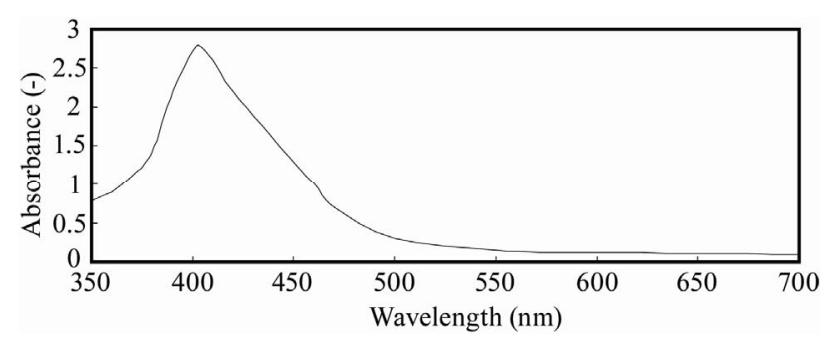

Figure 1. Absorption spectrum of silver nanoparticles prepared by solid-liquid extraction method.

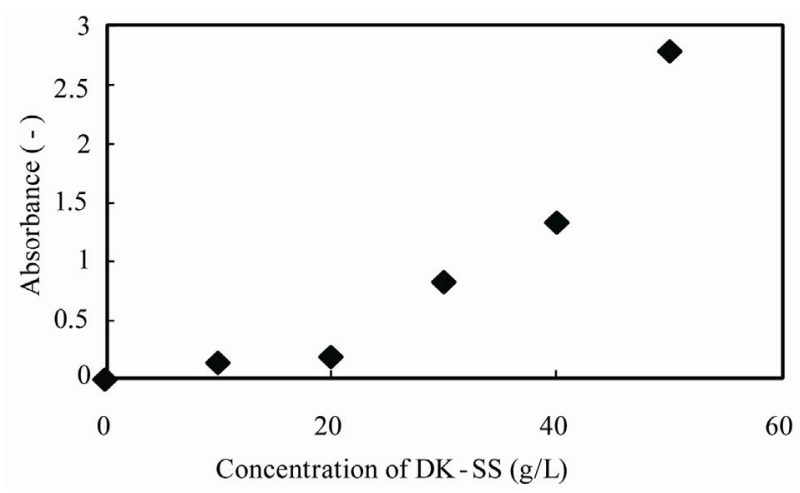

Figure 2. Effect of surfactant concentration on absorbance at $403 \mathrm{~nm}$ of colloidal solution dispersing silver nanoparticles prepared by solid-liquid extraction method: $\left[\mathrm{AgNO}_{3}\right]_{\mathrm{ov}}$ $=0.1 \mathrm{M} ;\left[\mathrm{NaBH}_{4}\right]_{\mathrm{ov}}=1.0 \mathrm{M}$; n-butanol/isooctane containing $50 \mathrm{~g} / \mathrm{L} \mathrm{DK}$-SS and $60 \mathrm{mM} \mathrm{H}_{2} \mathrm{O}$; reaction time $=3 \mathrm{~h}$; reaction temperature $=25^{\circ} \mathrm{C}$. tied reverse micelles approach solid reactants again. Thus, the production of silver nanoparticles is promoted by repeating the process composed of the extraction of reactants, the synthesis of silver nanoparticles, and the dispersion of resultant silver nanoparticles.

\subsection{Dependence of Reaction Temperature upon Size of Silver Nanopartices}

Figure 3 shows the typical transmission electron micrographs and size distributions of silver nanoparticles when silver nanoparticles were prepared in the reverse micellar system of DK-SS for $3 \mathrm{~h}$ at different reaction temperatures. The TEM images showed that the obtained silver nanoparticles displayed a wide variety of shapes. The size of resultant silver nanoparticles was almost similar to that prepared in DK-SS or AOT (sodium bis(2-ethylhexyl) sulfosuccinate) reverse micelles by the conventional injection method $[10,14,15]$. The size of silver nanoparticles was strongly dependent upon reaction temperature. Figure 4 shows the plots of the mean diameter of silver nanoparticles against reaction temperature. As seen in the figure, the mean diameter of silver nanoparticles gradually increased with an increase in reaction temperature. The line in the figure represents the fitting curve with the correlation coefficient of 0.99 as

$$
d=0.079 t+6.2
$$

where $d$ is the mean diameter of silver nanoparticles, and $t$ is the reaction temperature. This tendency was similar to the case using the conventional injection method [10]. The solubilization of reactants, the exchange of reactants between reverse micelles, the reduction reaction, and the growth of nanoparticles increase with increasing the reaction temperature [16-19].

Figure 5 shows the plots of the mean diameter of silver nanoparticles obtained at different reaction temperature against the wavelength in the peak of UV-visible absorption spectrum due to the LSPR of silver nanoparticles. The standard deviation concerning the size distribution of silver nanoparticles depended upon reaction temperature. The wavelength in the peaks increases with an increase in the mean diameter of silver nanoparticles. The line in the figure represents the fitting curve with the correlation coefficient of 0.99 as

$$
\lambda=8.2 d+340
$$

where $\lambda$ is the wavelength of peaks, and $d$ is the mean diameter of silver nanoparticles. The position of the plasmon adsorption peak depends upon the particle size and shape, and especially tends to be red-shifted with increasing the particle size [14,16,20,21]. 

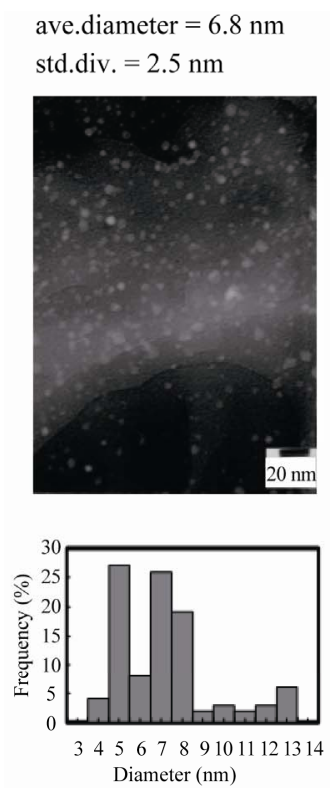

(a)

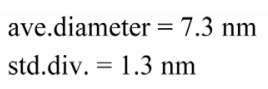

ave. diameter $=7.3 \mathrm{~nm}$ std.div. $=1.3 \mathrm{~nm}$
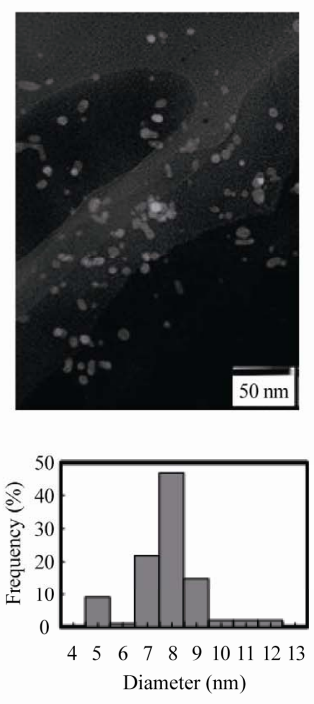

(b) ave. diameter $=7.8 \mathrm{~nm}$

std.div. $=2.0 \mathrm{~nm}$
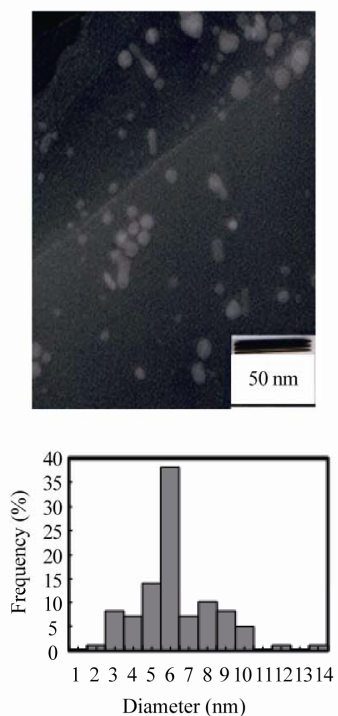

(c) ave. diameter $=9.6 \mathrm{~nm}$

std.div. $=2.5 \mathrm{~nm}$
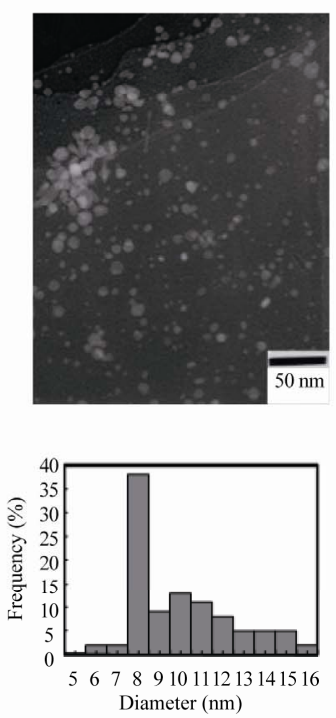

(d)

Figure 3. Transmission electron micrographs and particle size distributions of silver nanoparticles synthesized in DK-SS reverse micelles: reaction temperature $=\left(\right.$ a) $5^{\circ} \mathrm{C}$, (b) $15^{\circ} \mathrm{C}$, (c) $25^{\circ} \mathrm{C}$, and (d) $40^{\circ} \mathrm{C}$.

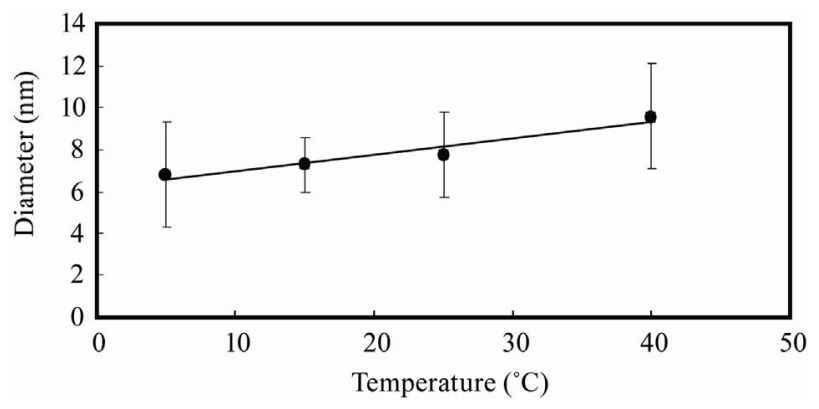

Figure 4. Effect of reaction temperature on the mean diameters of silver nanoparticles in reverse micelles of DK-SS: $\left[\mathrm{AgNO}_{3}\right]_{\mathrm{ov}}=0.1 \mathrm{M} ;\left[\mathrm{NaBH}_{4}\right]_{\mathrm{ov}}=1.0 \mathrm{M} ;$ n-butanol/isooctane containing $50 \mathrm{~g} / \mathrm{L}$ DK-SS and $60 \mathrm{mM} \mathrm{H} \mathrm{H}_{2} \mathrm{O}$; reaction time = $3 \mathbf{h}$.

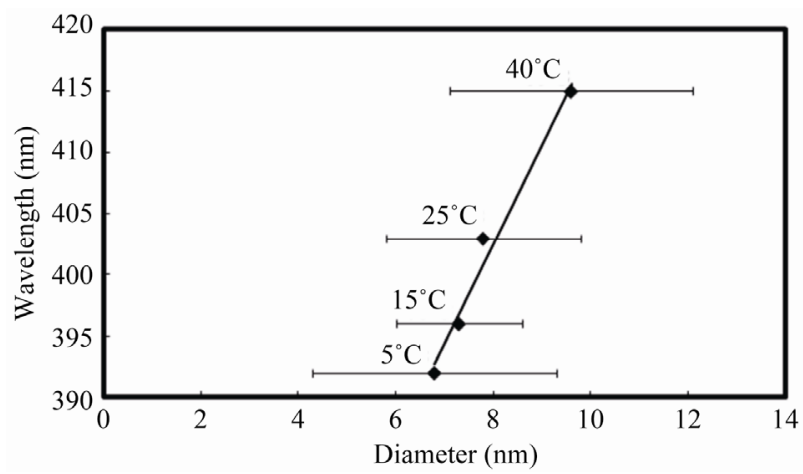

Figure 5. Relationship of the mean diameter of silver nanoparticles obtained at different reaction temperature with the wavelength in the peak of UV-visible absorption spectrum due to the LSPR of silver nanoparticles.

\subsection{Dependence of Average Esterification Degree of Surfactants upon Size of Silver Nanoparticles}

The formation and stability of micelles are due to the structure and/or HLB of surfactants [22-24]. The structure and HLB of sucrose fatty acid esters alter with the esterification degree [11]. In order to elucidate the effect of those factors on the formation of silver nanoparticles, we have examined the synthesis of silver nanoparticles in the reverse micelles of sucrose fatty acid esters by mixing DK-SS (average esterification degree $=1.01$, HLB $=$ 19 ) with DK-F-20W (average esterification degree $=3.1$, HLB = 2). The more weight fraction of DK-F-20W is, the larger the average esterification degree is. Figure 6 shows the typical transmission electron micrographs and size distributions of silver nanoparticles when silver nanoparticles were prepared in the reverse micellar system of sucrose fatty acid esters at different average esterification degrees for $3 \mathrm{~h}$. The silver nanoparticles in various shapes were obtained in those reverse micellar systems. The standard deviation concerning the size distribution of silver nanoparticles was strongly dependent upon the average esterification degree of surfactants. When the average esterification degree was 1.85 , the minimal standard deviation was obtained. This result indicated that at that average esterification degree, monodisperse silver nanoparticles were formed. As shown in Figure 7, the mean diameter of silver nanoparticles was dependent upon the average esterification degree, similar 

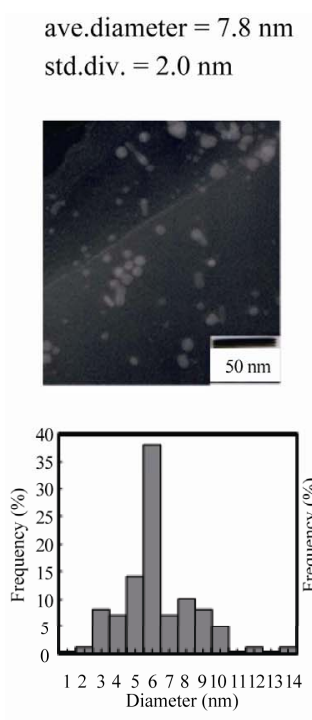

(a) ave. diameter $=7.9 \mathrm{~nm}$

std.div. $=1.9 \mathrm{~nm}$
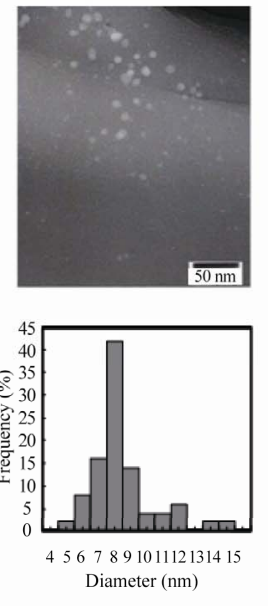

(b) ave.diameter $=8.2 \mathrm{~nm}$ std.div. $=1.6 \mathrm{~nm}$
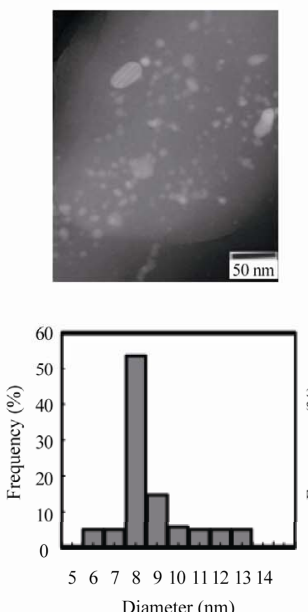

(c) ave. diameter $=9.2 \mathrm{~nm}$ std.div. $=1.8 \mathrm{~nm}$
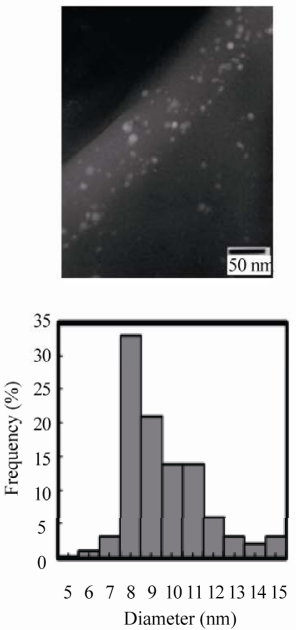

(d) ave. diameter $=12.4 \mathrm{~nm}$

std.div. $=3.1 \mathrm{~nm}$

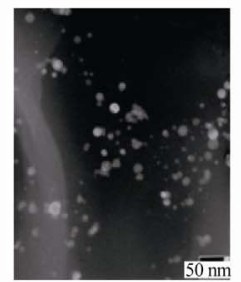

(e)

Figure 6. Transmission electron micrographs and particle size distributions of silver nanoparticles synthesized in reverse micelles of DK-SS and DK-F-20W: average esterification degree = (a) 1.01, (b) 1.43, (c) 1.85, (d) 2.68, and (e) 3.10.

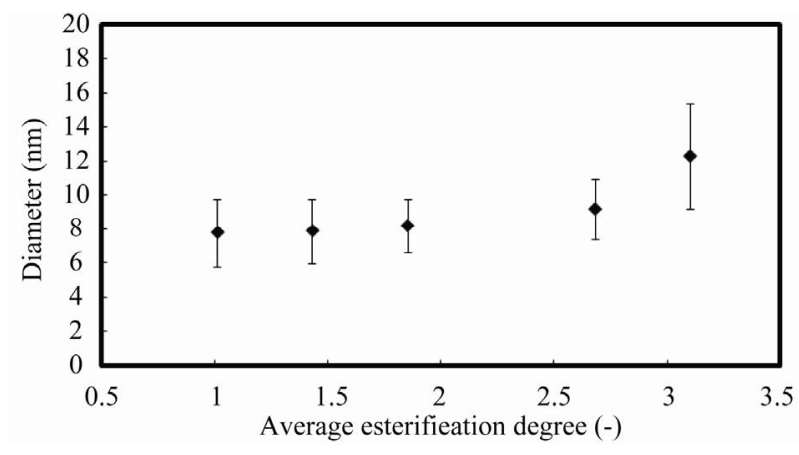

Figure 7. Effect of average esterification degree of surfactants on the size of silver nanoparticles synthesized in reverse micelles of DK-SS and DK-F-20W: $\left[\mathrm{AgNO}_{3}\right]_{\mathrm{ov}}=0.1 \mathrm{M}$; $\left[\mathrm{NaBH}_{4}\right]_{\text {ov }}=1.0 \mathrm{M}$; n-butanol/isooctane containing $50 \mathrm{~g} / \mathrm{L}$ DK-SS/DK-F-20W and $60 \mathrm{mM} \mathrm{H}_{2} \mathrm{O}$; reaction temperature $=25{ }^{\circ} \mathrm{C}$; reaction time $=3 \mathrm{~h}$.

to the case of injection method $[10,25]$. The size of silver nanoparticles gradually increased with an increase in the average esterification degree. Thus, when the bulkiness of surfactants increased or the HLB value decreased, the size of silver nanoparticles tended to increase.

\section{Conclusions}

We have demonstrated that the synthesis of silver nanoparticles is drastically promoted in the sucrose fatty acid ester/n-butanol/isooctane system by supplying reactants to the water pool through solid-liquid extraction. The size of silver nanoparticles decreased with a decrease in reaction temperature. The wavelength in the peaks corresponding upon the LSPR was linearly cor- related with the size of silver nanoparticles prepared by the present method. The size of silver nanoparticles tended to depend upon the average esterification degree of sucrose fatty acid esters.

\section{Acknowledgements}

Authors thank Mr. Misaki for taking TEM micrographs, and Dai-Ichi Kogyo Seiyaku Co., Ltd. for supplying sucrose fatty acid esters.

\section{References}

[1] J. H. Fendler, "Nanoparticles and Nanostructured Films: Preparation, Characterization and Applications," WileyVCH, Weinheim, 1998.

[2] J. Zhang, P. Chen, C. Sun and X. Hu, "Sonochemical Synthesis of Colloidal Silver Catalysts for Reduction of Complexing Silver in DTR System,” Applied Catalysis A: General, Vol. 266, No. 1, 2004, pp. 49-54. doi:10.1016/j.apcata.2004.01.025

[3] H. J. Lee, S. Y. Yeo and S. H. Jeong, “Antibacterial Effect of Nanosized Silver Colloidal Solution on Textile Fabrics," Journal of Materials Science, Vol. 38, No. 10, 2003, pp. 2199-2204. doi:10.1023/A:1023736416361

[4] Q. Zhang, Y. N. Tan, J. Xie and J. Y. Lee, "Colloidal Synthesis of Plasmonic Metallic Nanoparticles," Plasmonics, Vol. 4, No. 1, 2009, pp. 9-22. doi:10.1007/s11468-008-9067-X

[5] A. Sridhar, D. J. van Dijk and R. Akkerman, "Inkjet Printing and Adhesion Characterization of Conductive Tracks on a Commercial Printed Circuit Board Material," Thin Solid Films, Vol. 517, No. 16, 2009, pp. 4633-4637. 


\section{doi:10.1016/j.tsf.2009.03.133}

[6] J. Chen, Y. Luo, Y. Liang, J. Jiang, G. Shen and R. Yu, "Surface-Enhanced Raman Scattering for Immunoassay Based on the Biocatalytic Production of Silver Nanoparticles," Analytical Sciences, Vol. 25, No. 3, 2009, pp. 347-352. doi:10.2116/analsci.25.347

[7] V. Uskokovic and M. Drofenik, "Synthesis of Materials within Reverse Micelles," Surface Review and Letters, Vol. 12, No. 2, 2005, pp. 239-277. doi:10.1142/S0218625X05007001

[8] P. L. Luisi, "Enzymes Hosted in Reverse Micelles in Hydrocarbon Solution," Angewandte Chemie, Vol. 24, No. 6, 1985, pp. 439-528. doi:10.1002/anie.198504393

[9] A. Kitahara, T. Tamura and S. Matsumura, "Effect of Water on Stability of Carbon Black Dispersed in Nonaqueous Aerosol OT Solutions,” Chemistry Letters, Vol. 8, No. 9, 1979, pp. 1127-1128.

[10] H. Noritomi, N. Igari, K. Kagitani, Y. Umezawa, Y. Muratsubaki and S. Kato, "Synthesis and Size Control of Silver Nanoparticles Using Reverse Micelles of Sucrose Fatty Acid Esters,” Colloid and Polymer Science, Vol. 288, No. 8, 2010, pp. 887-891. doi:10.1007/s00396-010-2214-X

[11] N. Otomo, "Self-Organized Structures of Food Emulsifiers and Their Applications," Japan Journal of Food Engineering, Vol. 4, No. 1, 2003, pp. 1-9.

[12] A. Henglein, "Physicochemical Properties of Small Metal Particles in Solution: 'Microelectrode' Reactions, Chemisorption, Composite Metal Particles, and the Astom-toMetal Transition," Journal of Physical Chemistry, Vol. 97, No. 21, 1993, pp. 5457-5471. doi:10.1021/j100123a004

[13] H. Noritomi, S. Ito, N. Kojima, S. Kato and K. Nagahama, "Forward and Backward Extractions of Cytochrome C Using Reverse Micellar System of Sucrose Fatty Acid Ester," Colloid and Polymer Science, Vol. 284, No. 6, 2006, pp. 604-610. doi:10.1007/s00396-005-1398-y

[14] A. Taleb, C. Petit and M. P. Pileni, "Synthesis of Highly Monodisperse Silver Nanoparticles from AOT Reverse Micelles: A Way to 2D and 3D Self-Organization," Chemisty of Materials, Vol. 9, No. 4, 1997, pp. 950-959. doi:10.1021/cm960513y

[15] W. Zhang, X. Qiao, J. Chen and H. Wang, "Preparation of Silver Nanoparticles in Water-in-Oil AOT Reverse Micelles,” Journal of Colloid and Interface Science, Vol. 302, No. 1, 2006, pp. 370-373. doi:10.1016/j.jcis.2006.06.035

[16] R. P. Bagwe and K. C. Khilar, "Effects of Intermicellar Exchange Rate on the Formation of Silver Nanoparticles in Reverse Microemulsions of AOT," Langmuir, Vol. 16, No. 3, 2000, pp. 905-910. doi:10.1021/la980248q

[17] W. L. Hinze, "Organized Assemblies in Chemical Analysis Vol. 1: Reverse Micelles,” JAI Press, London, 1994.

[18] C. Tojo, M. C. Blanco and M. A. Lopez-Quintela, "The Influence of Reactant Excess and Film Flexibility on the Mechanism of Nanoparticle Formation in Microemulsions: A Monte Carlo Simulation,” Langmuir, Vol. 14, No. 24, 1998, pp. 6835-6839. doi:10.1021/la9806931

[19] J. P. Cason, M. E. Miller, J. B. Thompson and C. B. Roberts, "Solvent Effects on Copper Nanoparticle Growth Behavior in AOT Reverse Micelle Systems," Journal of Physical Chemistry B, Vol. 105, No. 12, 2001, pp. 22972302. doi:10.1021/jp002127g

[20] C. Petit, P. Lixon and M. P. Pileni, "In-Situ Synthesis of Silver Nanocluster in AOT Reverse Micelles,” Journal of Physical Chemistry, Vol. 97, No. 49, 1993, pp. 1297412983. doi:10.1021/j100151a054

[21] K. P. Charle, F. Frank and W. Schulze, "The Optical Properties of Silver Microcrystallites in Dependence on Size and the Influence of the Matrix Environment," Berichte der Bunsengesellschaft für physikalische Chemie, Vol. 88, No. 4, 1984, pp. 354-350.

[22] D. J. Shaw, "Introduction to Colloid and Surface Chemistry,” Butterworth-Heinemann, Oxford, 1992.

[23] J. N. Israelachvili, "Intermolecular and Surface Forces," Academic Press, London, 1985.

[24] D. J. Mitchell and B. W. Ninham, "Micelles, Vesicles, and Microemulsions," Journal of the Chemical Society, Faraday Transactions 2: Molecular and Chemical Physics, Vol. 77, No. 4, 1981, pp. 601-629.

[25] H. Noritomi, K. Kagitani, Y. Muratsubaki and S. Kato, "Effect of Composition of Sucrose Fatty Acid Esters on Formation of Palladium Nanoparticles in Reverse Micelles," Colloid and Polymer Science, Vol. 287, No. , 2009, pp. 795-799. 